\title{
A Label-Free Proteomic Strategy to Investigate the Intramuscular fat Proteomic Differences Between Biceps Femoris and Longissimus Dorsi in Inner Mongolian Cashmere Goats
}

\author{
Yuchun Xie \\ Inner Mongolia Agricultural University \\ Zhihong Liu \\ Inner Mongolia Agricultural University \\ Rile Nai \\ Hulunbuir University \\ Juntao Guo \\ Inner Mongolia Agriculture University

\section{Xin Su} \\ Inner Mongolia Agricultural University
}

Cun Zhao

Inner Monglia Agricultural University

Chongyan Zhang

Inner Mongolia Agricultural University

Qing Qin

Inner Mongolia Agricultural University

\section{Dongliang Dai}

Inner Mongolia Agricultural University

\section{Zhixin Wang}

Inner Mongolia Agricultural University

Yanhong Zhao

Inner Mongolia Agricultural University

Rui Su

Inner Mongolia Agricultural University

Zhiying Wang

Inner Mongolia Agricultural University

\section{Ruijun Wang}

Inner Mongolia Agricultural University

Yanjun Zhang

Inner Mongolia Agricultural University 
Jinquan Li ( $\nabla$ lijinquan_nd@126.com )

Inner Mongolia Agricultural University

Haijun Li

Inner Mongolia Agricultural University

\section{Research article}

Keywords: cashmere goat meat, IMF, anabolism and catabolism, label-free strategy, proteome

Posted Date: December 21st, 2020

DOl: https://doi.org/10.21203/rs.3.rs-131926/v1

License: (c) (i) This work is licensed under a Creative Commons Attribution 4.0 International License.

Read Full License 


\section{Abstract}

Background: As a major raw-cashmere-producing province in China. Nearly 700,000 Aerbasi cashmere goats are fed per year, and the corresponding meat production is nearly 10,000 tons. However, there are no reports on the meat of this goat. To better understand the molecular variations underlying intramuscular fat (IMF) anabolism and catabolism in Inner Mongolian cashmere goats, the proteomic differences between the biceps femoris (BF) and longissimus dorsi (LD) were investigated by a label-free strategy. Then, the identified proteins were verified as being involved in IMF anabolism and catabolism by Western blot analysis.

Results: The IMF content was significantly higher in the BF than in the LD, suggesting that IMF accumulated more in the BF or was metabolized more in the LD. We performed proteomic analysis of IMF anabolism and catabolism at the proteomic level, and 1209 proteins were identified in the BF (high-IMF) and LD (low-IMF) groups. Among them, 110 were differentially expressed proteins (DEPs), 81 of which were upregulated in the high-IMF group, while 29 were upregulated in the low-IMF group. Gene ontology (GO) classification showed that the 110 DEPs were functionally classified into 100 annotation clusters. Kyoto Encyclopedia of Genes and Genomes (KEGG) analysis showed that the 110 DEPs covered 34 KEGG pathways. Three pathways were related to IMF metabolism and deposition-fatty acid metabolism, fatty acid degradation and fatty acid elongation-and included 7 proteins.

Conclusion: GO and KEGG analyses showed that differentially expressed HADHA, HADHB, ACSL1, ACADS, ACAT 1 and ACAA2 in the mitochondria act via fatty acid metabolism, fatty acid degradation and fatty acid elongation to influence the metabolism and synthesis of long-, short- and medium-chain fatty acids and modulate IMF anabolism and catabolism. Protein-protein interaction (PPI) network analysis showed that IMF accumulation in different muscle tissues of Inner Mongolian cashmere goats was affected not only by 5 key enzymes and proteins involved in fatty acid synthesis and metabolism but also by five DEPs (SUCLG1, SUCLG2, CS, DLST, and ACO2) in the TCA cycle. Our results provide new insights into IMF deposition in goats and improve our understanding of the molecular mechanisms underlying IMF anabolism and catabolism.

\section{Background}

As a major raw-cashmere-producing province in China, Inner Mongolia cashmere goats harbors three types -Erlangshan, Aerbasi and Alashan [1]. The Inner Mongolian cashmere goat is a local breed that provides both cashmere and meat, and the production of cashmere in Inner Mongolia accounts for approximately $40 \%$ of the total output of the whole country [2]. Nearly 700,000 Aerbasi cashmere goats are fed per year, and the corresponding meat production is nearly 10,000 tons. Aerbasi cashmere has been widely studied, but the meat value of this goat has not been reported. Therefore, the study of Aerbasi cashmere goat meat is of great significance. 
Intramuscular fat (IMF) is mainly found in the sarcolemma, including the perimysium, epimysium and endomysium, and is mainly composed of triglycerides and phospholipids [3]. The IMF content and its fatty acid composition play an important role in meat quality, affecting the sensory properties (flavor, juiciness and tenderness) and nutritional value of meat [4]. The positive effect of IMF on the sensory quality of meat has been demonstrated in pork [5], mutton [6], and beef [7]. The IMF deposition capacity is regulated by 3 aspects: transport to fatty acids, fat anabolism and fat catabolism. Recent studies have demonstrated that $\mathrm{FABP}$ and $\mathrm{CD}_{36}$ play important roles in the process of fat uptake $[8,9]$, and $\mathrm{DGAT}_{1}$ and SCD regulate the synthesis of IMF $[10,11]$. The HSL and LPL genes regulate fat breakdown $[12,13]$. However, it is not known which proteins affect the deposition and metabolism of IMF in cashmere goat meat, so research on the composition of protein in cashmere goat meat is urgently needed.

In recent years, the label-free approach to proteomics, which is known to be a reliable, versatile, and costeffective strategy, has received much attention [14]. Furthermore, it has been successfully applied to compare proteome changes in beef color stability [15] and gain knowledge of broiler self-regulation mechanisms under heat stress [16]. However, the application of a label-free proteomics strategy to investigate proteomic changes that occur during the process of IMF regulation has not yet been reported in cashmere goats. Moreover, the proteomic abundance in cashmere goats remains unclear. Therefore, the objective of the present study was to identify key proteins involved in IMF anabolism and catabolism and to reveal underlying biochemical events with the help of bioinformatics analyses.

\section{Result}

\section{IMF content of the LD and BF muscles}

To assess the IMF content, the longissimus dorsi (LD) and biceps femoris (BF) tissues were collected from 20 goats. Independent sample t-tests for fatty acids in different muscles showed that the IMF content was significantly higher in the BF than in the LD $(P<0.01)$ (Fig. 1). This suggests that IMF is deposited at higher levels in the BF or metabolized to a greater degree in the LD. To explore IMF anabolism and catabolism at the proteomic level, we selected the BF and LD from 6 goats for the next experiment.

\section{Protein Identification And Comparative Analysis}

To understand the protein composition of these six goats, the UniProt/Swiss-Prot/Capra hircus database was used as a reference to investigate the proteome during IMF anabolism and catabolism using a labelfree mass spectrometry strategy. A total of 1209 proteins were identified in the BF (high-IMF group) and LD (low-IMF group), and 993 and 896 proteins were identified in each group with a false discovery rate $(F D R) \leq 0.01$ (supplementary material 1). Therefore, there are many proteins that are yet to be studied in Inner Mongolian cashmere goat. 
To visualize and differentiate the observed sample clusters, Principal component analysis (PCA) was performed to compare the high-IMF group and low-IMF group based on the proteins present in both groups. PCA is an unsupervised method that can condense a large number of variables (proteins) into a set of representative and uncorrelated principal components by means of their variance-covariance structure. The score plot of the PCA of the high-IMF group and low-IMF group (Fig. 2a) showed that $45.9 \%$ of the variability was explained by the first two principal components, which accounted for $31.8 \%$ and $14.5 \%$ of the total variance. Samples from the two groups could be separated completely and were located in different quadrants, which indicated the existence of differentially abundant proteins between the high-IMF group and low-IMF group.

The volcano plots present samples with fold changes $>2$ or $<0.5$ and P-value $<0.05$ (Fig. 2b). In the comparison of the high-IMF group and low-IMF group, there were a total of 110 significant differentially expressed proteins (DEPs) that were associated with the accumulation or metabolism of IMF; 81 proteins were upregulated in the high-IMF group, whereas 29 proteins were upregulated in the low-IMF group. The detailed results for the DEPs are presented in supplementary material 2.

\section{Function Analysis Of Differentially Expressed Proteins}

According to the Gene ontology (GO) classification statistics (Fig. 3), DEPs from IMF metabolism and deposition can be divided into three main categories: biological processes, cell components, and molecular functions. In this study, the 110 DEPs were functionally classified into 100 annotation clusters (supplementary material 3). The top 10 annotated GO terms in the biological process category showed that the DEPs participated in the oxidation-reduction process, small molecule metabolic process, oxoacid metabolic process, organic acid metabolic process, generation of precursor metabolites and energy, and carboxylic acid metabolic process (Fig. 3a). The top $10 \mathrm{GO}$ terms in the cellular component category indicated that the DEPs were mainly enriched in mitochondria, the intracellular region, organelles, intracellular organelles, the cytoplasm, and the mitochondrial envelope. The top $10 \mathrm{GO}$ terms in the molecular function category indicated that the DEPs were mainly involved in catalytic activity, ion binding, oxidoreductase activity, cytoskeletal protein binding, and actin binding.

It is speculated that during IMF metabolism and deposition, the increase or decrease in proteins or enzymes leads to changes in the metabolic rate or accumulation, which further leads to differences in the IMF content.

Kyoto Encyclopedia of Genes and Genomes (KEGG) analysis was performed to evaluate the potential functions of exclusively detected proteins and DEPs. The top 20 enriched KEGG terms of these proteins are described in Fig. $3 \mathrm{~b}$. The results show that the 110 investigated proteins cover 34 KEGG pathways. In total, three of the pathways were related to IMF metabolism and deposition, including fatty acid metabolism, fatty acid degradation and fatty acid elongation. Seven proteins were included in these three pathways, such as acetyl-CoA acyltransferase $2\left(\mathrm{ACAA}_{2}\right)$, cholesterol acyltransferase $1\left(\mathrm{ACAT}_{1}\right)$, and 
Enoyl-CoA hydratase (HADHA) (Supplementary material 3). The KEGG analysis results provide further insight into IMF anabolism and catabolism.

Protein-protein interaction (PPI) network analyses were performed to construct the specific molecular network involving the key DEPs related to IMF anabolism and catabolism between the high-IMF group and the low-IMF group.

To search for hub proteins among the DEPs, we used the software CytoHubba. One PPI network was determined, covering 33 DEPs and 147 edges (Fig. 4). In addition, the top 10 DEPs in the network were identified, as ranked by MCC method. The results showed that the 33 DEPs cooperated to form an IMF anabolism and catabolism PPI network centered on $\mathrm{ACAT}_{1}, \mathrm{ACAA}_{2}$, Succinate-CoA ligase [ADP/GDPforming] subunit alpha (SUCLG $)_{1}$, Succinate-CoA ligase [GDP-forming] subunit beta $\left(S U C L G_{2}\right)$, Trifunctional enzyme subunit beta (HADHB), Citrate synthase (CS), Dihydrolipolylysine-residue succinyltransferase component of 2-oxoglutarate dehydrogenase complex (DLST), Enoyl-CoA hydratase $\left(E_{C H S}\right)$, Aconitate hydratase $\left(\mathrm{ACO}_{2}\right)$, and HADHA. Among these 10 hub proteins, $\mathrm{ACAT}_{1}$ and $\mathrm{ACAA}_{2}$ targeted or were targeted by another 9 proteins; $S U C L G_{1}$ and $S U C L G_{2}$ targeted or were targeted by another 8 proteins (except HADHA); HADHB targeted or was targeted by another 8 proteins (except $\mathrm{ACO}_{2}$ ); $\mathrm{CS}$ and DLST targeted or were targeted by another 7 proteins (except HADHA and ECHS1); ECHS ${ }_{1}$ targeted or was targeted by another 7 proteins (except CS and DLST); ACO2 targeted or was targeted by another 7 proteins (except HADHA and HADHB); and HADHA targeted or was targeted by another 5 proteins (except DLST, SUCLG 1, SUCLG 2 and $A C C_{2}$ ). These 10 hub genes play a vital role in regulating IMF anabolism and catabolism.

Both functional analysis and PPI network analysis showed that HADHA, ACAA 2 and $\mathrm{ACAT}_{1}$ might play an important role in regulating IMF anabolism and catabolism in Inner Mongolian cashmere goat.

\section{Protein Determination By Western Blot Analysis}

Protein determination by Western blot analysis

Western blot quantification is a critical method that provides accurate and reproducible results (Fig. 5). Therefore, this method was used to detect the expression levels of three DEPs (HADHA, fatty acid-binding protein $\left(\mathrm{FABP}_{3}\right)$ and $A M P$-binding domain-containing protein $\left.\left(\mathrm{ACS}_{1}\right)\right)$ from the high-IMF group and lowIMF group. All three proteins were upregulated in the high-IMF groups. The average band intensities of $\mathrm{HADHA}, \mathrm{FABP}_{3}$ and $\mathrm{ACSL}_{1}$ (normalized to $\beta$-tubulin) had similar variation tendencies according to the results of MS analyses. The band intensities of these three proteins were significantly higher in the BF than in the LD $(p<0.05)$.

\section{Discussion}


IMF is formed by the deposition of fat in muscle, which is composed of IMF and myofibrils. A previous study showed that the IMF content ultimately depends on fatty acid transport, IMF anabolism and IMF catabolism $[17,18]$. The process of fatty acid transport involves fatty acids entering intramuscular cells to provide the necessary substrates for IMF synthesis; IMF anabolism includes the synthesis, elongation or desaturation of fatty acid chains and the synthesis of triglycerides; IMF catabolism includes mobilization of fat in intramuscular cells and hydrolysis of triglycerides in lipoproteins. Interestingly, in our study, we found that the IMF content was significantly higher in the BF than in the LD. This suggests that IMF is deposited at greater levels in the BF or metabolized to a greater degree in the LD. This provides a good model for studying the regulatory mechanisms of IMF anabolism and catabolism.

IMF anabolism and catabolism are closely associated with many critical cellular functions and biological processes. For example, IMF or fatty acid triglycerides are metabolized in the mitochondrial matrix, and this process is called fatty acid beta-oxidation [19]. Fatty acid beta-oxidation generally includes four steps: oxidation, hydration, oxidation and cleavage [20]. In our study, the GO classification statistics of 110 DEPs showed that the top biological process was the oxidation-reduction process, which included 36 DEPs. In the cellular component analysis, the most enriched cellular component was the mitochondrion, which included 39 DEPs. In the molecular function analysis, 65 DEPs were mainly enriched in catalytic activity. Overall, the DEPs were mostly located in mitochondria, had catalytic activity, and participated in oxidation processes. In summary, the GO analysis results were consistent with the function of fatty acid beta-oxidation steps, and DEPs involved in these processes were the key proteins contributing to the differences in fatty acid beta-oxidation, further influencing IMF metabolism.

The KEGG enrichment analyses indicated that there were 3 terms (fatty acid metabolism, fatty acid degradation and fatty acid elongation) related to IMF, and 7 of the DEPs were associated with these three pathways. The seven proteins included in these three pathways were $\mathrm{ACAA}_{2}$, short-chain acyl-coenzyme $A$ dehydrogenase (ACADS), ACAT ${ }_{1}, \mathrm{ACSL}_{1}$, short-chain enoyl-CoA hydratase $\left(\mathrm{ECHS}_{1}\right), \mathrm{HADHA}$, and HADHB. Fatty acids can be classified as long-chain (containing more than 12 carbon atoms), medium- -chain (containing 6-12 carbon atoms) and short-chain (containing less than 6 carbon atoms) fatty acids [21]. Short-chain fatty acids can directly cross the outer mitochondrial membrane and enter the mitochondrial matrix for fatty acid oxidation, but long-chain and medium-chain fatty acids need to be transported through the inner mitochondrial membrane under the catalysis of carnitine acyltransferase 1 , which is located on the outer mitochondrial membrane. Among the 7 DEPs enriched by KEGG analysis, ACSL1, $\mathrm{HADHA}$ and $\mathrm{HADHB}$ are mitochondrial membrane proteins that act on long-chain fatty acids. $\mathrm{ACSL}_{1}$ is known to catalyze the first step (oxidation) of the activation of long-chain fatty acids by converting them into long-chain acyl-CoA thioesters for channeling toward chain elongation, triacylglyceride synthesis or fatty acid oxidation [22]. ACSL ${ }_{1}$ is necessary for the synthesis of long-chain acyl-CoA esters, fatty acid degradation and phospholipid remodeling [23]. HADHA and HADHB, which break down fatty acids to acetyl-CoA, are specific for long-chain fatty acids. HADHA is involved in fatty acid beta-oxidation, which is a part of lipid metabolism. Research has shown that HADHA overexpression significantly inhibits cell growth, induces cell apoptosis, and decreases the formation of cytoplasmic lipid droplets [24]. ACADS is 
one of the acyl-CoA dehydrogenases that catalyze the first step (oxidation) of mitochondrial fatty acid beta-oxidation, an aerobic process that breaks down fatty acids to acetyl-CoA. It acts specifically on acylCoAs with saturated 4- to 6-carbon-long primary chains. Studies have shown that ACADS not only plays a vital role in free fatty acid $\beta$-oxidation but also regulates energy homeostasis [25]. $\mathrm{ECHS}_{1}$, similar to ACADS in function, also participates in the metabolism of fatty acyl coenzyme $A$ ester and is an important mitochondrial fatty acid beta oxidase but acts on straight-chain enoyl-CoA thioesters that have 4 to at least 16 carbons [26]. ACAT 1 and $\mathrm{ACAA}_{2}$ are involved in lipid metabolism. They are two key enzymes of the fatty acid oxidation pathway, catalyzing the last step (cleavage) of mitochondrial betaoxidation. They use free coenzyme $\mathrm{A} / \mathrm{CoA}$ and catalyze the thiolytic cleavage of medium- to long-chain unbranched 3-oxoacyl-CoAs to acetyl-CoA and fatty acyl-CoA, which are shorter by two carbon atoms, thus playing an important role in fatty acid metabolism [27]. GO and KEGG analyses showed that in the mitochondrion differentially expressed HADHA, HADHB and $\mathrm{ACSL}_{1}$ participate in the fatty acid metabolism, fatty acid degradation and fatty acid elongation pathways to influence long-chain fatty acid metabolism and synthesis, further influencing IMF anabolism and catabolism. Differentially expressed ACADS affects fatty acid metabolism, fatty acid degradation and fatty acid elongation, influencing shortchain fatty acid metabolism and synthesis and further influencing IMF anabolism and catabolism; Differentially expressed $A C A T_{1}$ and $A C A A_{2}$ act on fatty acid metabolism, fatty acid degradation and fatty acid elongation, influencing long-chain and medium-long-chain fatty acid metabolism and synthesis and further influencing IMF anabolism and catabolism.

In the construction of the PPI network, a total of 10 hub proteins were found. Among them, $A C A T_{1}, A_{C A A_{2}}$, $\mathrm{HADHB}, \mathrm{ECHS}_{1}$, and HADHA were also identified by GO and KEGG analyses, and the other 5 interacting proteins were $\mathrm{SUCLG}_{1}, \mathrm{SUCLG}_{2}, \mathrm{CS}, \mathrm{DLST}$, and $\mathrm{ACO}_{2}$. All five of these proteins are involved in the tricarboxylic acid cycle (TCA). Succinate-CoA ligase (SUCL) is a heterodimer consisting of an alpha subunit encoded by $S U C L G_{1}$ and a beta subunit encoded by $S U C L G_{2}$, catalyzing an ATP- and a GTPforming reaction, respectively $[28,29]$. SUCL is at the intersection of several metabolic pathways [30]. For example, SUCLA 2 rebound increases, pleiotropically affecting metabolic pathways associated with SUCL [31]. CS synthesizes isocitrate from oxaloacetate, and Tereza Škorpilová, et al showed that there could be specific limits designed for CS activity in chilled and frozen/thawed meats [32]. DLST catalyzes the overall conversion of 2-oxoglutarate to succinyl-CoA and $\mathrm{CO}_{2}$ [33]. $\mathrm{ACO}_{2}$ catalyzes the isomerization of citrate to isocitrate, and $\mathrm{AcO} 2$ is needed for mitochondrial translation [34]. Analysis of the PPI network showed that in addition to the 5 key enzymes and proteins involved in the process of fatty acid synthesis and metabolism affecting the final accumulation of IMF in different muscle tissues of Inner Mongolian cashmere goat, there are also five proteins in the TCA cycle that exhibit expression, thereby affecting the accumulation of IMF.

In our study, the proteomic profiles of the high-IMF and low-IMF muscles of Inner Mongolia cashmere goats were evaluated. Our results provide new insights into IMF deposition in goats and improve our understanding of the molecular mechanisms associated with IMF anabolism and catabolism. 
Conclusion: GO and KEGG analysis results show that differentially expressed HADHA, HADHB, $\mathrm{ACSL}_{1}$, ACADS, ACAT 1 and $A C A A_{2}$ in mitochondria act on fatty acid metabolism, fatty acid degradation and fatty acid elongation pathways to influence long-chain fatty acid, short-chain fatty acid and medium-chain fatty acid metabolism and synthesis and to influence IMF anabolism and catabolism. PPI network analysis results showed that IMF accumulation in different muscle tissues of Inner Mongolian cashmere goats was affected not only by 5 key enzymes and proteins involved in the process of fatty acid synthesis and metabolism but also by five DEPs $\left(\mathrm{SUCLG}_{1}, \mathrm{SUCLG}_{2}, \mathrm{CS}, \mathrm{DLST}, \mathrm{ACO}{ }_{2}\right)$ involved in the TCA cycle. Our results provide new insights into IMF deposition in goats and improve our understanding of the molecular mechanisms associated with IMF anabolism and catabolism.

\section{Method}

\section{Animals and sample collection}

The BF and LD were sampled from each animal (two years old, wether, carcass weight of $25-26 \mathrm{~kg}$ ) as 6 replicate samples, all of which were of Inner Mongolia cashmere goats (Aerbasi, Yiwei White Cashmere Goat Breeding Farm, Erdos, Inner Mongolia). The experiments were approved by the experimental animal ethics committee of Inner Mongolia Agricultural University (GB 14925-2001). And permission were obtained from the farm owner. Animals were slaughtered under controlled conditions after being electrically stunned, and then, the muscles were collected aseptically into enzyme-free tubes, immediately immersed in liquid nitrogen and subsequently stored at $-80^{\circ} \mathrm{C}$ until analysis.

\section{IMF content of meat}

The IMF level for the BF and LD was determined using the Soxhlet extraction protocol following the method of Hopkins et al. [35]. Three grams of freeze-dried meat was weighed into a thimble and extracted in $85 \mathrm{~mL}$ of hexane for 60 minutes within individual extraction tins. The solvent was then evaporated off for an additional 20 minutes. The tin was then dried for 30 minutes at $105^{\circ} \mathrm{C}$ to remove any residual solvent. The variation in meat weight before and after extraction was used to calculate IMF content. The final value is expressed as a percentage of meat weight.

\section{Protein extraction and digestion}

Each frozen sample was ground to powder in liquid nitrogen and dissolved in lysis buffer (proteinase inhibitors (Roche, Basel, Switzerland), 1\% SDS (Coolaber, China)). The samples were then incubated at room temperature for 20 minutes, vortexing for 30 seconds every minute. After 20 minutes of ultrasonication, the samples were centrifuged at $12,000 \mathrm{rpm}$ and $4^{\circ} \mathrm{C}$ for 30 minutes. The supernatant was collected for further study, and the protein concentration was measured with a bicinchoninic acid (BCA) kit (Tiangen, China).

One hundred micrograms of protein was added to $200 \mu \mathrm{L}$ of $8 \mathrm{M}$ urea (Sigma, Germany) and $10 \mathrm{mM} \mathrm{DL}-$ dithiothreitol (Sigma-Aldrich, Germany) and incubated at $37^{\circ} \mathrm{C}$ for 1 hour. After centrifugation at 12,000 
rpm for 40 minutes, $200 \mu \mathrm{L}$ of urea was added to each filtrate tube, which was then agitated. Next, the filtrate tubes were centrifuged twice at 12,000 rpm for 30 minutes each. Then, $200 \mu \mathrm{L}$ of $50 \mathrm{mM}$ iodoacetamide (Sigma-Aldrich, Germany) was added to each filtrate tube; the reaction was allowed to proceed in the dark for 30 minutes, and then, the liquid was removed. Next, $100 \mu \mathrm{L}$ of ammonium bicarbonate (Fluka, Germany) was added to each filtrate tube, and the samples were centrifuged at $12,000 \mathrm{rpm}$ for 20 minutes. This step was performed 3 times, and then, the liquid was removed. The samples were incubated overnight with trypsin at $37^{\circ} \mathrm{C}$ and centrifuged at $12,000 \mathrm{rpm}$ for 30 minutes. Then, $50 \mu \mathrm{L}$ of ammonium bicarbonate (Fluka, Germany) was added to each filtrate tube. The samples were centrifuged at $12,000 \mathrm{rpm}$ for 30 minutes, and this step was repeated. The filtrate was collected, freeze-dried, and stored at $-20^{\circ} \mathrm{C}[36]$.

\section{HPLC-MS/MS analysis}

Two methods, namely, information-dependent acquisition (IDA) and sequential window acquisition of all theoretical fragment ion spectra (SWATH), were used to acquire data from separated peptides on the LCMS/MS system (Sciex, Framingham, MA, USA). Approximately $2 \mu \mathrm{g}$ of peptides was injected and separated on a C18 HPLC column $(75 \mu \mathrm{m} \times 15 \mathrm{~cm})$. A linear gradient $(120$ minutes, going from 5 to $80 \% \mathrm{~B}$ at $500 \mathrm{~nL} /$ minute) of $0.1 \%$ formic acid in water and $0.1 \%$ formic acid in acetonitrile was used to separate peptides. The conditions for IDA were as follows: nominal resolving power of 30,000, time-of-flight (TOF)MS collection from 350 to $1800 \mathrm{~m} / \mathrm{z}$ and automated collision energy for MS/MS with IDA scanned from 400 to $1800 \mathrm{~m} / \mathrm{z}$. The conditions for SWATH-MS were as follows: 150-1200 m/z MS1 mass range, 100$1500 \mathrm{~m} / \mathrm{z}$ MS2 spectra, and nominal resolving power of 30,000 and 15,000 for MS1 and MS2, respectively.

\section{Data processing}

Protein Pilot 4.5 software (Sciex, Framingham, MA, USA) was used with the UniProt/SWISS-PROT (https://www.UniProt.org/\#) database (downloaded from https://www.UniProt.org; 556,388 proteins) to identify peptides. The results were filtered at a $1 \%$ FDR. The selected search parameters included the use of trypsin as the enzyme, allowing up to two missed cleavage sites. The peptide mass tolerance was \pm 15 ppm, and the fragment mass tolerance was $20 \mathrm{mmu}$. The data were loaded into PeakView (Sciex, Framingham, MA, USA) software to search the SWATH databank using the ion library generated in Protein Pilot. PeakView generated extracted ion chromatograms (XICs) after processing targeted and nontargeted data. Then, the results were interpreted and quantitatively analyzed using MarkerView software (Sciex, Framingham, MA, USA). MarkerView allows a rapid review of data to determine the DEPs. PCA and volcano plot analysis, which combined fold change analysis and t-tests, were performed. A fold change $>2$ or fold change $<0.5$ and statistical significance ( $p$ value $<0.05$ ) were used to identify DEPs [37].

\section{Bioinformatics analysis}


The DEPs were subjected to bioinformatics analyses. g:Profiler (https://biit.cs.ut.ee/gprofiler/gost) online software was used to perform the GO and KEGG pathway analyses. Cytoscape_v3.6.1 was used to perform the PPI analysis of DEPs, and cytoHubba was used for screening hub genes [38].

\section{Western blotting}

The homogenized denatured protein was separated by SDS-PAGE and transferred in a semidry state to a PVDF membrane (Bio-Rad, USA). The PVDF membrane was blocked in blocking buffer (Li-CoR, USA) for 2 hours and incubated overnight with murine monoclonal primary antibody (Abcam, Germany). The membrane was washed 3 times for 10 minutes each time and then incubated with fluorophoreconjugated goat anti-mouse antibody (Li-CoR, USA) for 1 hour. Finally, the membrane was rinsed with water, and the immunoreactive bands were examined using a LI-COR Odyssey (CLX-0496, USA) nearinfrared imager.

\section{Abbreviations}

BF: biceps femoris; LD: longissimus dorsi; DEPs: differentially expressed proteins; IMF: intramuscular fat; ACAA2: acyltransferase 2; ACAT1: cholesterol acyltransferase 1; HADHA: Enoyl-CoA hydratase; SUCLG1: succinate-CoA ligase [ADP/GDP-forming] subunit alpha; SUCLG2: Succinate--CoA ligase [GDP-forming] subunit beta; HADHB: trifunctional enzyme subunit beta; CS: citrate synthase; DLST: dihydrolipolylysineresidue succinyltransferase component of 2-oxoglutarate dehydrogenase complex; ECHS1: enoyl-CoA hydratase; ACO2: aconitate hydratase; FABP3: fatty acid-binding protein; ACSL1: AMP-binding domaincontaining protein; ACADS: short-chain acyl-coenzyme A dehydrogenase; ECHS1: short-chain enoyl-CoA hydratase

\section{Declarations}

\section{Ethics approval and consent to participate}

Samples were collected in accordance with the Guidelines for Experimental Animals of the Ministry of Science and Technology (Beijing, China) and were approved by the experimental animal ethics committee of Inner Mongolia Agricultural University (GB 14925-2001).

\section{Consent for publication}

All authors agree to publish.

\section{Availability of data and materials}

The mass spectrometry proteomics data have been deposited to the ProteomeXchange Consortium (http://proteomecentral.proteomexchange.org) via the iProX partner repository with the dataset identifier PXD022901. 


\section{Competing interests}

The authors declare that they have no competing interests.

\section{Funding}

This work received funding from the National Key Research and Development Program (2018YFD0502000), the Major Science and Technology Projects of the Inner Mongolia Autonomous Region (2020ZD0004), The National Natural Science Foundation of China (31660640), The National Natural Science Foundation of China (32060742), and the Key Technology Project of the Inner Mongolia Autonomous Region (2020GG0030). The funding bodies played no role in the design of the study, collection, analysis, and interpretation of data or in the writing of the manuscript.

\section{Author contributions}

$Y X, Z L$ and RN made substantial contributions to the conception and design of the experiments. Conception and design of experiments: $Y X, Z L, R N, J G, X S, C Z, C Z$ Authors $Y X, R N, Z W, X S, Q Q$, and DD performed the experiments. Authors $Y X, Z L, R N, C Z, Y Z, R S$ and $H L$ analyzed the data. Authors $Y X, Z L, Z W$, $\mathrm{RW}$ and $\mathrm{CZ}$ wrote the paper, and authors $\mathrm{JL}$ and $\mathrm{HL}$ critically revised the manuscript. All authors read and approved the final manuscript.

\section{Acknowledgements}

The authors are thankful for the samples provided by the Aerbasi White Cashmere Goat Breeding Farm. Jinquan Li provided the test platform, and the co-first authors of this article, Zhihong Liu and Haijun Li helped in designing and conducting the experiments and in analyzing, evaluating and interpreting the results.

\section{Authors' information}

College of Animal Science, Inner Mongolia Agricultural University, Hohhot, 010018, China.

Key Laboratory of Animal Genetics, Breeding and Reproduction in the Inner Mongolia Autonomous Region

Key Laboratory of Mutton Sheep Genetics and Breeding of the Ministry of Agriculture

The Inner Mongolia Autonomous Region Goat Genetics and Breeding Engineering Technology Research Center

School of Agriculture and Forestry, Hulunbuir University, Hulunbuir 021008, China

College of Veterinary Medicine, Inner Mongolia Agricultural University, Hohhot 010018, China 


\section{References}

1. Su R, Fan Y, Qiao X, Li X, Zhang L, Li C, Li J. Transcriptomic analysis reveals critical genes for the hair follicle of Inner Mongolia cashmere goat from catagen to telogen. PLoS One. 2018 Oct;24;13(10):e0204404.

2. Su R, Gong G, Zhang L, Yan X, Wang F, Zhang L, Qiao X, Li X, Li J. Screening the key genes of hair follicle growth cycle in Inner Mongolian Cashmere goat based on RNA sequencing. Arch Anim Breed. 2020 May;26;63(1):155-64.

3. GUO B, KONGSUWAN K, GREENWOOD PL, et al. A gene expression estimator of intramuscular fat percentage for use in both cattle and sheep. Journal of Animal Science Biotechnology. 2014;4:37990.

4. Hocquette JF, Gondret F, Baéza E, Médale F, Jurie C, Pethick DW. Intramuscular fat content in meatproducing animals: development, genetic and nutritional control, and identification of putative markers. Animal. 2010 Feb;4(2):303-19.

5. Gao SZ, Zhao SM. Physiology, affecting factors and strategies for control of pig meat intramuscular fat. Recent Pat Food Nutr Agric. 2009 Jan;1(1):59-74.

6. Watkins PJ, Frank D, Singh TK, Young OA, Warner RD. Sheepmeat flavor and the effect of different feeding systems: a review. J Agric Food Chem. 2013 Apr;17;61(15):3561-79.

7. Mateescu RG, Garrick DJ, Garmyn AJ, VanOverbeke DL, Mafi GG, Reecy JM. Genetic parameters for sensory traits in longissimus muscle and their associations with tenderness, marbling score, and intramuscular fat in Angus cattle. J Anim Sci. 2015 Jan;93(1):21-7.

8. He J, Chen J, Lu L, Tian Y, Tao Z, Wang D, Li J, Li G, Shen J, Fu Y, Niu D. A novel SNP of liver-type fatty acid-binding protein gene in duck and its associations with the intramuscular fat. Mol Biol Rep. 2012 Feb;39(2):1073-7.

9. YANG Y, SONG J, FU R Q, et al. AMPK subunit expression regulates intramuscular fat content and muscle fiber type in chickens. Agricultural Science $11 \% 4 \rrbracket$ Technology. 2015;16(5):1006-10.

10. Anton I, Kovács K, Holló G, et al. Effect of leptin, DGAT1 and TG gene polymorphisms on the intramuscular fat of Angus cattle in Hungary. Livestock ence. 2011;135(2-3):300-3.

11. AVILS C, HORCADA A, POLVILLO O, et al. Association study between variability in the SCD gene and the fatty acid profile in perirenal and intramuscular fat deposits from Spanish goat populations. Small Ruminant Research 136(2016):127-131.

12. QIAO Y, HUANG Z G, LI Q F, et al. Developmental changes of the FAS and HSL mRNA expression and their effects on the content of intramuscular fat in Kazak and Xinjiang sheep. Journal of Genetics Genomics. 2007;34(10):909-17.

13. WANG W, XUE W, ZHOU X, et al. Effects of candidate genes'polymorphisms on meat quality traits in pigs. Acta Agriculturae Scandinavica, Section A: Animal Science 2012, 62(3): 120-126.

14. Gallego M, Mora L, Aristoy M, Concepción, et al. Optimisation of a simple and reliable label-free methodology for the relative quantitation of raw pork meat proteins. Food Chemistry 182(2015):74- 
80.

15. Yu Q, Wu W, Tian X, et al. Comparative proteomics to reveal muscle-specific beef color stability of Holstein cattle during post-mortem storage. Food Chem. 2017;229(AUG.15):769-78.

16. Tang X, Meng Q, Gao J, Zhang S, Zhang H, Zhang M. Label-free Quantitative Analysis of Changes in Broiler Liver Proteins under Heat Stress using SWATH-MS Technology. Sci Rep. 2015;5:15119.

17. Moczulski D, Majak I, Mamczur D. An overview of beta-oxidation disorders. Postepy Hig Med Dosw (Online). 2009 Jun 8;63:266-77.

18. Heeren J, Scheja L. Brown adipose tissue and lipid metabolism. Curr Opin Lipidol. 2018 Jun;29(3):180-5.

19. Rogers GW, Nadanaciva S, Swiss R, Divakaruni AS, Will Y. Assessment of fatty acid beta oxidation in cells and isolated mitochondria. Curr Protoc Toxicol. 2014 May;27(1):3.1-19.. ;60.

20. Zhang Y, Wang Y, Wang X, Ji Y, Cheng S, Wang M, Zhang C, Yu X, Zhao R, Zhang W, Jin J, Li T, Zuo Q, $\mathrm{Li}$ B. Acetyl-coenzyme A acyltransferase 2 promote the differentiation of sheep precursor adipocytes into adipocytes. J Cell Biochem. 2018 Nov 28.

21. Tvrzicka E, Kremmyda LS, Stankova B, Zak A. Fatty acids as biocompounds: their role in human metabolism, health and disease-a review. Part 1: classification, dietary sources and biological functions. Biomed Pap Med Fac Univ Palacky Olomouc Czech Repub. 2011 Jun;155(2):117-30.

22. Mashek DG, Li LO, Coleman RA. Long-chain acyl-CoA synthetases and fatty acid channeling. Future Lipidol. 2007;2:465-76.

23. Widmann P, Nuernberg K, Kuehn C, Weikard R. Association of an ACSL1 gene variant with polyunsaturated fatty acids in bovine skeletal muscle. BMC Genet. 2011 Nov 11;12:96.

24. Liu S, Liu X, Wu F, Zhang X, Zhang H, Gao D, Bi D, Qu H, Ge J, Xu Y, Zhao Z. HADHA overexpression disrupts lipid metabolism and inhibits tumor growth in clear cell renal cell carcinoma. Exp Cell Res. 2019 Nov;384(1)(1):111558.

25. Chen Y, Su Z. Reveal genes functionally associated with ACADS by a network study. Gene. 2015 Sep;15;569(2):294-302.

26. Sharpe AJ, McKenzie M. Mitochondrial Fatty Acid Oxidation Disorders Associated with Short-Chain Enoyl-CoA Hydratase (ECHS1) Deficiency. Cells. 2018 May 23;7(6):46.

27. Shaw L, Engel PC. The purification and properties of ox liver short-chain acyl-CoA dehydrogenase. Biochem J. 1984 Mar;1;218(2):511-20.

28. Chinopoulos C, Batzios S, van den Heuvel LP, Rodenburg R, Smeets R, Waterham HR, Turkenburg M, Ruiter JP, Wanders RJA, Doczi J, Horvath G, Dobolyi A, Vargiami E, Wevers RA, Zafeiriou D. Mutated SUCLG1 causes mislocalization of SUCLG2 protein, morphological alterations of mitochondria and an early-onset severe neurometabolic disorder. Mol Genet Metab. 2019 Jan;126(1):43-52.

29. Li X, Wu F, Beard D. Identification of the kinetic mechanism of succinyl-CoA synthetase. Bioence Reports. 2013;33(1):145-63. 
30. Tretter L, Patocs A, Chinopoulos C. Succinate, an intermediate in metabolism, signal transduction, ROS, hypoxia, and tumorigenesis. Biochim Biophys Acta 2016 Aug;1857(8):1086-1101.

31. Kacso G, Ravasz D, Doczi J, Németh B, Madgar O, Saada A, llin P, Miller C, Ostergaard E, lordanov I, Adams D, Vargedo Z, Araki M, Araki K, Nakahara M, Ito H, Gál A, Molnár MJ, Nagy Z, Patocs A, AdamVizi V, Chinopoulos C. Two transgenic mouse models for $\beta$-subunit components of succinate-CoA ligase yielding pleiotropic metabolic alterations. Biochem J. 2016 Oct;15;473(20):3463-85.

32. Škorpilová T, Šístková I, Adamcová M, Pohůnek V, Kružík V, Ševčík R. Measuring citrate synthase activity as an enzymatic approach to the differentiation of chilled and frozen/thawed meat. Meat Sci. 2019 Dec;158:107856.

33. Koike K, Suematsu T, Ehara M. Cloning, overexpression and mutagenesis of cDNA encoding dihydrolipoamide succinyltransferase component of the porcine 2-oxoglutarate dehydrogenase complex. Eur J Biochem. 2000 May;267(10):3005-16.

34. Jung SJ, Seo Y, Lee KC, Lee D, Roe JH. Essential function of Aco2, a fusion protein of aconitase and mitochondrial ribosomal protein bL21, in mitochondrial translation in fission yeast. FEBS Lett. 2015 Mar;24(7):822-8.. ;589.

35. Hopkins DL, Clayton EH, Lamb TA, et al. The impact of supplementing lambs with algae on growth, meat traits and oxidative status. Meat ence. 2014;98(2):135-41.

36. Wu W, Dai R, Bendixen. Emøke. Comparing SRM and SWATH Methods for Quantitation of Bovine Muscle Proteomes. J Agric Food Chem. 2019 Feb;6;67(5):1608-18.

37. Ebhardt HA, Degen S, Tadini V, et al. Comprehensive proteome analysis of human skeletal muscle in cachexia and sarcopenia: a pilot study. J Cachexia Sarcopenia Muscle 2017 Aug;8(4):567-582.

38. Yu EM, Zhang HF, Li ZF, et al. Proteomic signature of muscle fibre hyperplasia in response to faba bean intake in grass carp. entific Reports. 2017;7:45950.

\section{Figures}




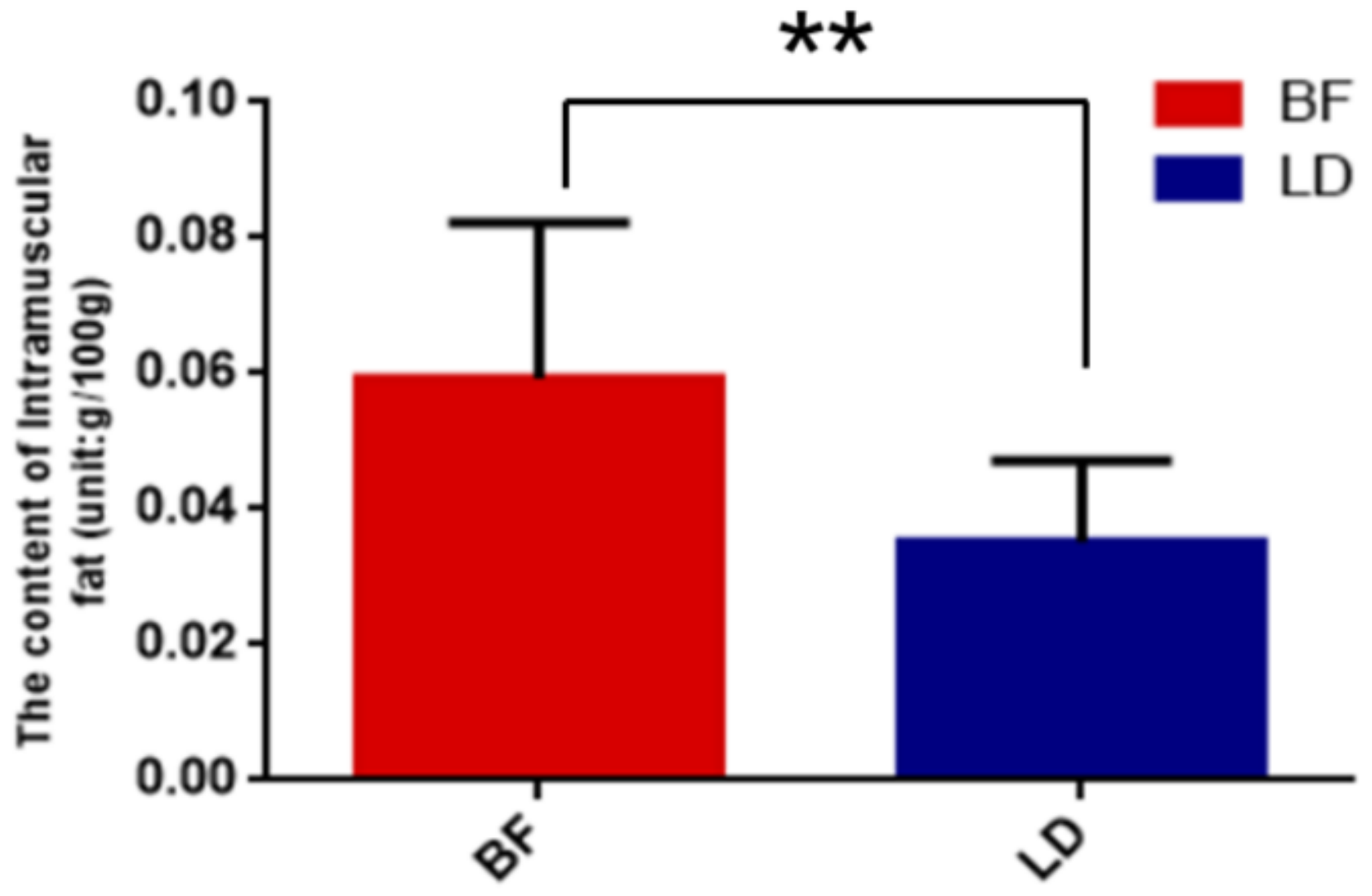

Figure 1

IMF content of the BF and LD muscle tissues. The BF and LD comprised the high-IMF and low-IMF groups, respectively. (t-test: $\left.{ }^{\star} \mathrm{p}<0.01\right)$.

a

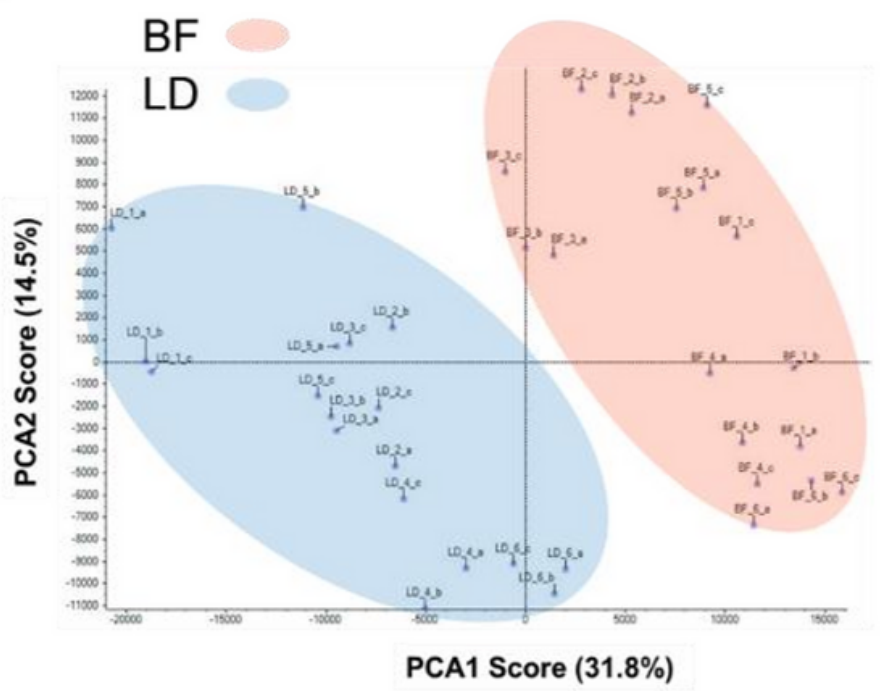

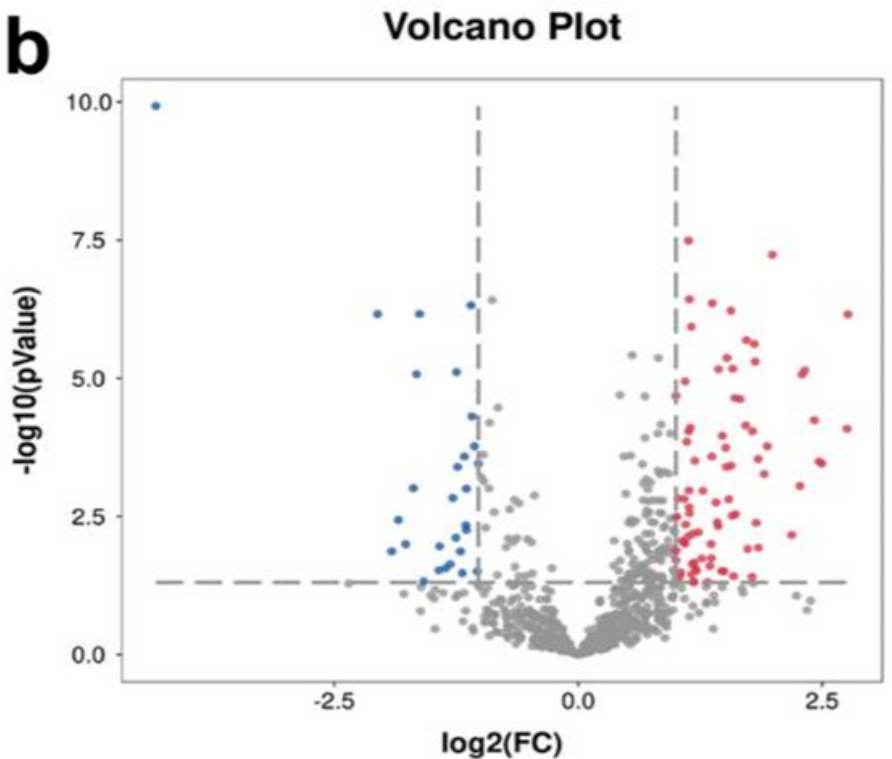

Figure 2 
Differentially expressed proteins identified. a: The PCA score plots of proteins in the high-IMF (red) and low-IMF groups (blue). b: The volcano plots show the comparison of the high-IMF and low-IMF groups, in which the points in red located in the right plots represent the proteins that were overabundant in the highIMF group with a fold-change of $2.0(p<0.05)$, while those in blue in the left plots represent the proteins that were overabundant in the low-IMF group.

a

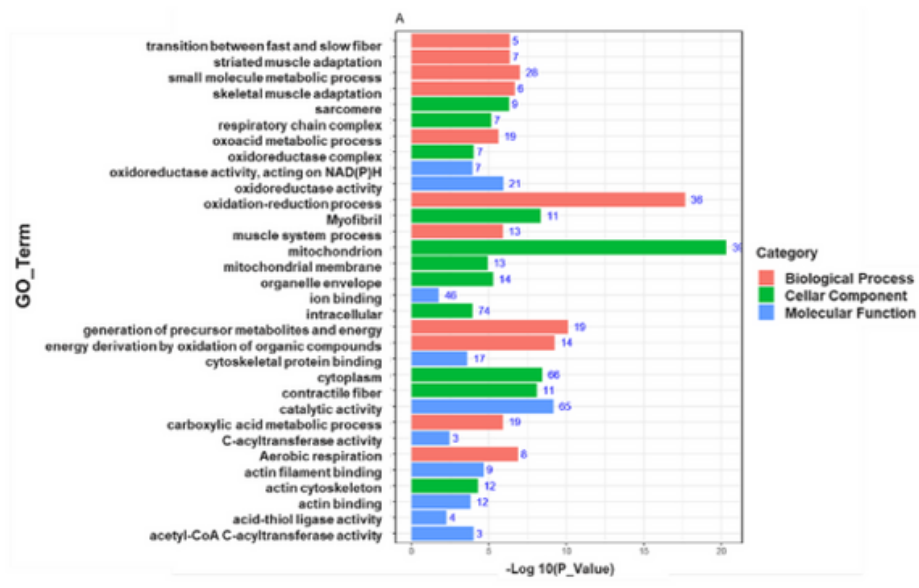

b

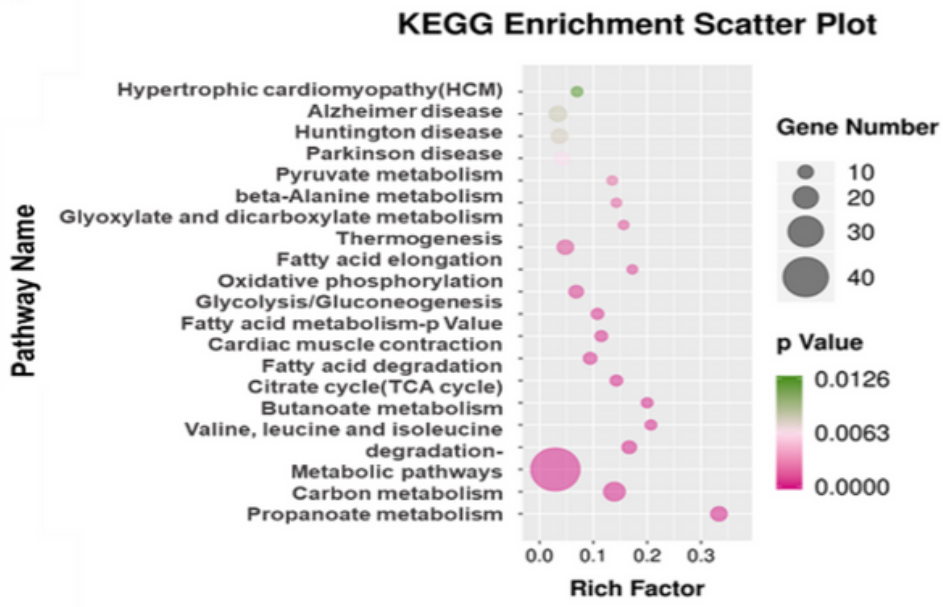

Figure 3

Differentially expressed proteins in skeletal muscle of the high-IMF and low-IMF groups. a. GO analysis. The numbers in blue represent DEPs. b. KEGG pathway analysis. Only the top 20 enriched pathways are presented here. 


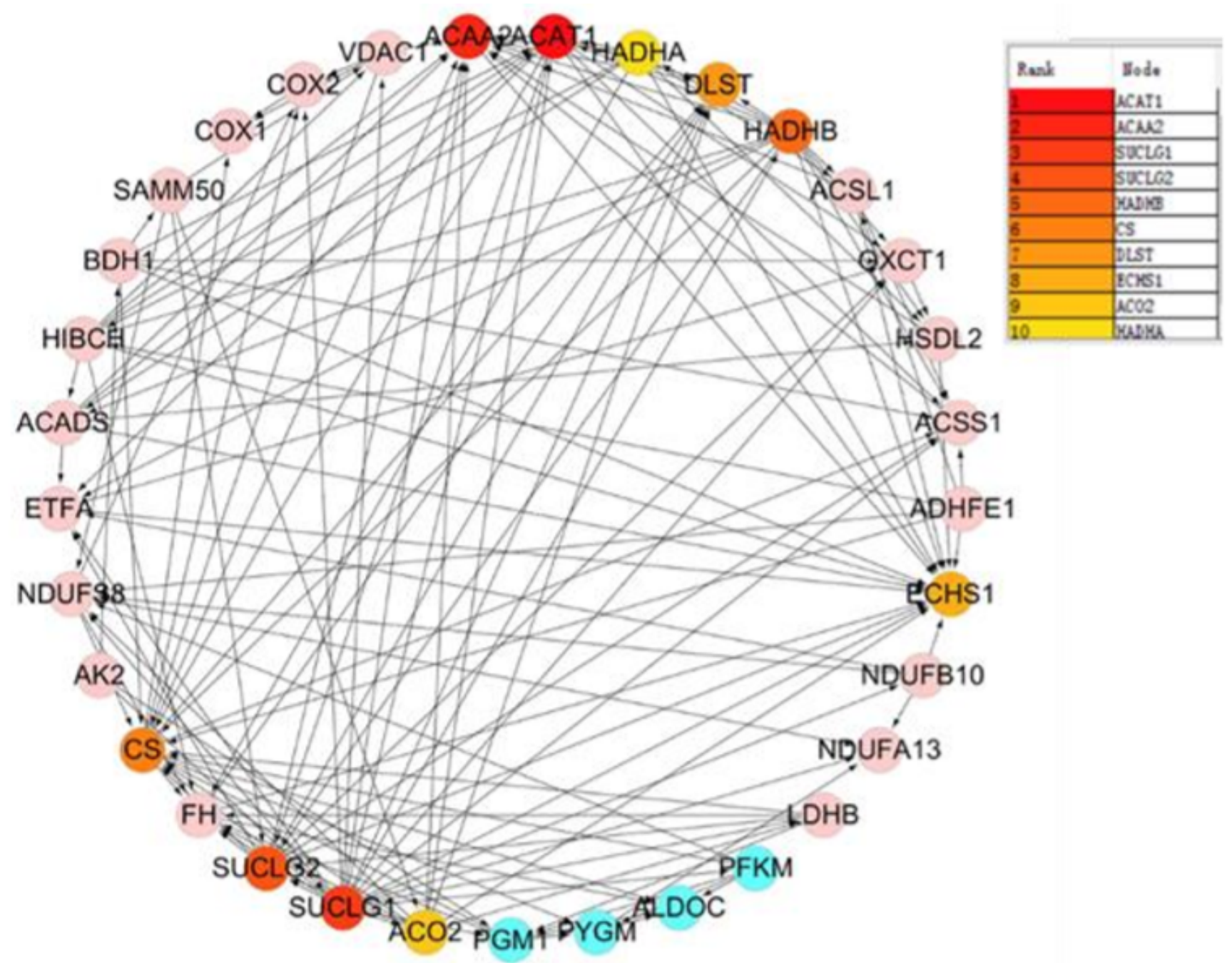

Figure 4

"See the Supplemental Files section for the complete figure caption".

\section{LD1 LD2 LD3 BF1 BF2 BF3}
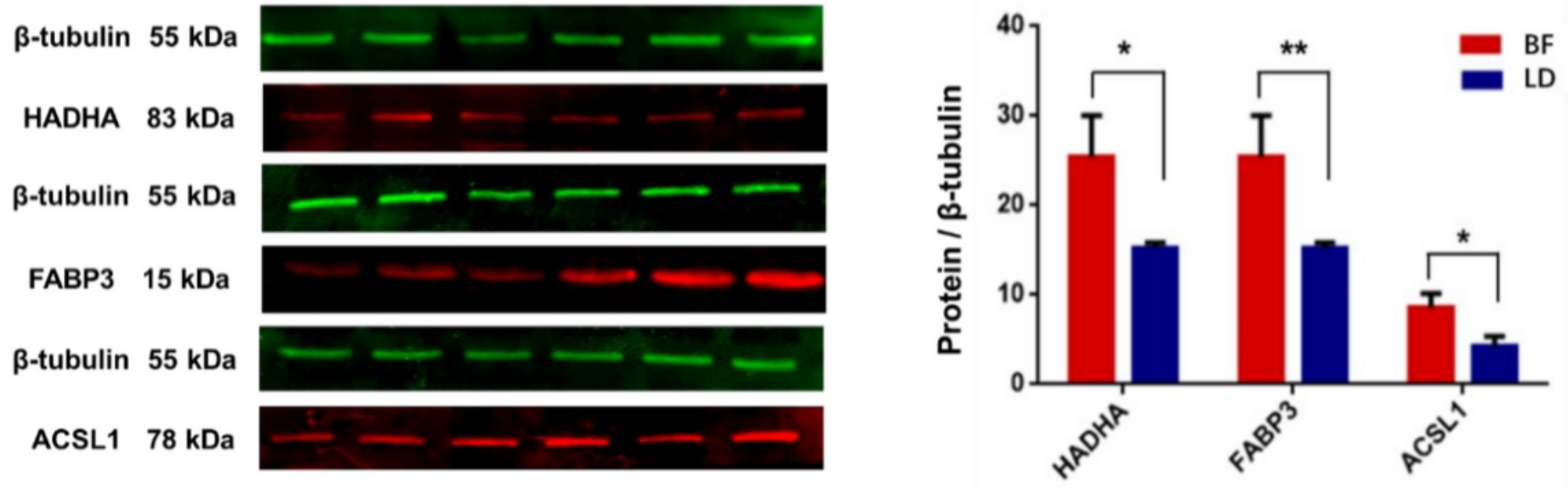

Figure 5 
Western blot profiles of ACSL1, HADHA, and FABP3 in the BF and LD. Three biological replicates from tissue of the BF and LD. Relative protein intensity is expressed as the mean \pm standard deviation. Asterisks represent levels of significance (t-test: $\left.{ }^{\star} p<0.05,{ }^{* \star} p<0.01\right)$.

\section{Supplementary Files}

This is a list of supplementary files associated with this preprint. Click to download.

- Fig4Caption.docx

- SupplementaryMaterial1Detectedprotein.xIsx

- SupplementaryMaterial2Diffrentiallyexpressedprotein.xlsx

- SupplementaryMaterial3FunctionanotionofDEPs.xlsx

- additionalfileofblotimages.pdf

- arrive.docx 